\title{
Asociación entre dopamina sérica y colesterol total en caninos
}

\author{
ASSOCIATION BETWEEN SERUM DOPAMINE AND SERUM TOTAL CHOLESTEROL IN DOGS
}

\section{Gonzalo Chávez C. ${ }^{1,3}$, Mauricio Maturana E. ${ }^{1}$, Claudia Rojas Z. ${ }^{1}$, Francisco Cuevas C. ${ }^{2}$}

\section{Resumen}

Se investigó la relación entre el colesterol total ( $\left.\mathrm{Col}_{\mathrm{t}}\right)$ y la dopamina (DA) a fin de disponer de un examen accesible en consulta como es la medición del colesterol total $\left(\mathrm{Col}_{\mathrm{t}}\right)$ de manera de proyectar el comportamiento de la DA sérica. Se determinó el Col a 50 caninos y sobre la base de los resultados se formaron tres grupos estudio, Grupo I: 6 pacientes con Col $_{\mathrm{t}}$ entre 77 y $200 \mathrm{mg} / \mathrm{dl}$; Grupo II: 6 pacientes entre 250 y $300 \mathrm{mg} / \mathrm{dl}$; Grupo III: 6 pacientes entre 400 y $470 \mathrm{mg} / \mathrm{dl}$. A los 18 pacientes se les midió la DA sérica a través de HPLC acoplado a un detector electroquímico. Se encontró una amplia distribución de

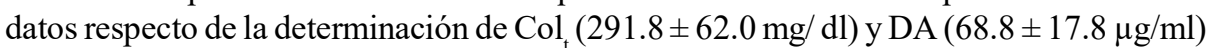
con una baja correlación entre ellos. El análisis por grupo de estudio muestra una mayor correlación de DA para bajas concentraciones de colesterol sérico, y el análisis de pendientes muestra que la variación en la concentración de DA es mayor ante pequeñas variaciones de colesterol en el grupo II.

Palabras clave: dopamina; colesterol; etología clínica; agresividad; perros

\section{AbStract}

The relationship between total cholesterol ( $\mathrm{Col}_{\mathrm{t}}$ ) and dopamine (DA) was investigated for the possibility of having an accessible exam in consultation such as the measurement of total cholesterol $\left(\mathrm{Col}_{t}\right)$ to project the behavior of serum DA. The $\mathrm{Col}_{t}$ was determined in 50 dogs and based on the results three study groups were formed, Group I: 6 patients with $\mathrm{Col}_{\mathrm{t}}$ between 77 and $200 \mathrm{mg} / \mathrm{dl}$; Group II: 6 patients between 250 and $300 \mathrm{mg} / \mathrm{dl}$;

${ }^{1}$ Escuela de Medicina Veterinaria, Facultad de Recursos Naturales y Medicina Veterinaria, Universidad Santo Tomás, Viña del Mar, Chile

${ }^{2}$ Departamento de Ciencias Básicas, Facultad de Ciencias, Universidad Santo Tomás, Viña del Mar, Chile

${ }^{3}$ E-mail: gchavez@santotomas.cl

Recibido: 20 de julio de 2017

Aceptado para publicación: 28 de diciembre de 2017 
Group III: 6 patients between 400 and $470 \mathrm{mg} / \mathrm{dl}$. Serum DA was measured in the 18 patients through HPLC coupled to an electrochemical detector. It was found a wide distribution of data regarding the determination of Col $(291.8 \pm 62.0 \mathrm{mg} / \mathrm{dl})$ and DA $(68.8$ $\pm 17.8 \mu \mathrm{g} / \mathrm{ml}$ ) with a low correlation between them. The analysis by study group shows a greater correlation of DA for low concentrations of serum cholesterol, and the analysis of slopes shows that the variation in the concentration of DA is greater in cases of small variations of cholesterol in group II.

Key words: dopamine; cholesterol; clinical ethology; aggressiveness; dogs

\section{INTRODUCCIÓN}

Una de las principales causas que debilitan el vínculo humano-animal son las conductas agresivas de los animales hacia el hombre. Por esta razón, se han realizado diversas investigaciones sobre las posibles causas que favorecen y modulan este tipo de conductas, identificándose varios posibles orígenes; como, por ejemplo, la relación entre el colesterol (Col) y la agresión (Pentürk y Yalçin, 2003; Pereira, 2017), así como entre la dopamina (DA) y la agresión (Miczek et al., 2002). Ambas han sido estudiadas individualmente, resultando de interés relacionar el Col con la DA, ya que la información al respecto es limitada. De existir dependencia, permitiría disponer de un examen accesible en consulta como es la medición del colesterol total $\left(\mathrm{Col}_{\mathrm{t}}\right)$ para proyectar, de este modo, el comportamiento de la DA sérica.

El Col es el tercer tipo de lípido en importancia cuantitativa en las membranas de las células animales, donde contribuye al mantenimiento de la fluidez de membrana y establece interacciones con ciertas proteínas de membrana que pueden regular su actividad (Allen et al., 2007). El Col se sintetiza a partir de acetil CoA a través de una serie de reacciones enzimáticas, donde la segunda fuente de Col celular ocurre a través de la captación de Col asociado a las lipoproteínas (Maldonado et al., 2012; Vega-Badillo, 2017). Del mismo modo, ayuda al metabolismo de los ácidos de la bilis, de las hormonas esteroides y de la síntesis de vitamina D (Kirk y Bonagura, 2001). Además, se debe considerar que existe un importante contenido de Col en las estructuras mielinizadas del cerebro y sistema nervioso central. Por otro lado, en relación con la DA, se puede mencionar que existen dos tipos de monoaminas: las catecolaminas y las indolaminas. Las primeras (norepinefrina, epinefrina y DA) son sintetizadas a partir del aminoácido tirosina (Murray et al., 2003), mientras que las indolaminas se sintetizan desde el triptófano (Goodman et al., 2007). La implicancia de las monoaminas sobre la conducta agresiva ha sido corroborada, tanto en estudios experimentales como clínicos (Goodman et al., 2007).

Las neuronas dopaminérgicas forman un sistema neurotransmisor, el cual se origina en la sustancia negra pars compacta, el área tegmental ventral y el hipotálamo. Esto proyecta axones a grandes partes del cerebro mediante cuatro vías (Dodman y Shuster, 1998), donde dos de ellas, la mesolímbica y la mesocortical, están implicadas en el desarro1 lo de severos desórdenes cognitivos y comportamentales (Lindsay, 2000). El aumento de la actividad dopaminérgica en el cerebro a través de sus múltiples receptores produce un incremento de las respuestas agresivas ante los estímulos del medio, interviniendo tanto en la iniciación, como en la ejecución del comportamiento agresivo (Miczek et al., 2002; Siever, 2008). Este neurotransmisor, también se relaciona con el aprendizaje, la memoria, el refuerzo y la motivación (Jackson y Westlind-Danielsson, 1994). 
Según Simons e Ikonen (1997), el receptor del transportador activo de DA $\left(\mathrm{DA}_{\mathrm{T}}\right)$ es una proteína integral de membrana de múltiples tramos incrustados en la bicapa lipídica plasmática, que comprende tres componentes principales: fosfolípidos, esfingolípidos y Col. Se han propuesto dominios ricos en Col dentro de la membrana para servir como una plataforma dinámica para la compartimentación y la organización de proteínas de membrana (Simons e Ikonen, 1997; Brown y London, 1998). Investigaciones sobre transportadores de serotonina, norepinefrina, glicina y aminoácidos excitatorios indican que pueden estar asociados con los dominios de membrana ricos en Col en los tejidos del cerebro o líneas celulares transfectadas (Butchbach et al., 2004; Jayanthi et al., 2004; Magnani et al., 2004; Núñez et al., 2008). La actividad de transporte de sustrato de estos carriers se inhibe en general por el agotamiento del Col. La asociación del receptor $\mathrm{DA}_{\mathrm{T}}$ con dominios de membrana ricos en $\mathrm{Col}$ se ha propuesto para regular el tráfico y la movilidad lateral de $\mathrm{DA}_{\mathrm{T}}$ en la membrana (Adkins et al., 2007; Foster et al., 2008). Asimismo, se ha demostrado que en microdominios de membrana ricos en Col, los $\mathrm{DA}_{\mathrm{T}}$ adoptan una conformación tal que aumenta la liberación de DA (Hong y Amara, 2010). Sin embargo, no existen estudios relacionados con el contenido de colesterol plasmático y su posible relación con los niveles de DA. En este sentido el presente trabajo pretende determinar si existe esta posible relación en una población canina.

\section{Materiales y Métodos}

En una primera fase, se trabajó con 50 perros en la Región de Valparaíso continental, Chile, entre junio y noviembre de 2015. Los pacientes fueron reclutados por estudiantes de la Escuela de Medicina Veterinaria de la Universidad Santo Tomás, Viña del Mar. Los criterios de inclusión fueron: condición corporal $3 / 5$, entre 1 y 5 años, intactos, sin alteraciones de conducta y alimentados con alimento comercial certificado por la Association of American Feed Control Officials. El sexo del animal no fue excluyente y el tipo de conducta del animal fue determinado por un médico veterinario certificado en etología clínica por el Colegio Latinoamericano de Etología Clínica Veterinaria. Por otro lado, los criterios de exclusión fueron estar en tratamiento farmacológico, presentar alguna enfermedad metabólica o estar gestante.

Luego de un ayuno de 12 horas, se colectó $5 \mathrm{ml}$ de sangre venosa y se determinó el $\mathrm{Col}_{\mathrm{t}}$ sérico mediante una prueba colorimétrica de bioquímica húmeda, con base a un reactivo enzimático Cholesterol liquicolor (Human, Alemania) y utilizando el analizador enzimático Humastar $80 \AA$ (Human, Alemania). Se realizaron curvas de calibrado y cuantificación de límites de detección que dieron como resultado valores similares a los reportados por el kit. De cada muestra de sangre se congeló suero para que, en caso de seleccionarse al paciente, se midiera DA en un plazo no mayor a un mes.

En una segunda fase, se seleccionaron 18 individuos entre los 50 perros iniciales, quienes fueron distribuidos en tres grupos, según el resultado de $\mathrm{Col}_{\mathrm{t}}$ : Grupo 1, seis pacientes con los valores más bajos (77$200 \mathrm{mg} / \mathrm{dl}$ ); Grupo 2, seis pacientes con los valores centrales (250-300 mg/dl); Grupo 3, seis pacientes con los valores más altos (400$470 \mathrm{mg} / \mathrm{dl}$ ). Se consideraron como valores de referencia de normalidad aquellos entre 135 y $345 \mathrm{mg} / \mathrm{dl}$ (Wittwer, 2012).

El trabajo experimental in situ se llevó a cabo en el Hospital Escuela Veterinario de la Universidad Santo Tomás, Viña del Mar, Chile. En los 18 perros del estudio se determinó la DA sérica en el Laboratorio de Análisis Clínico de la Pontificia Universidad Católica de Valparaíso con el uso de cromatografía líquida de alta presión (HPLC) acoplada a un detector electroquímico. Para la medición, las muestras fueron despro- 
teinizadas a través de la adición de tricloro acético al $10 \%$ y luego centrifugada a $1020 \mathrm{~g}$ por $5 \mathrm{~min}$. Los resultados obtenidos fueron sometidos a pruebas de evaluación de normalidad D'Agostino-Pearson y de ShapiroWilk a fin de realizar estadística paramétrica. Para determinar si existía alguna relación entre las variables se realizó una prueba de linealidad determinando el coeficiente de correlación lineal de Pearson.

Los propietarios de los animales incluidos en el estudio firmaron una carta de consentimiento informado donde se explicaba el propósito del estudio, las etapas, las consecuencias para el animal y se dejaba explícito que podían retirarse del mismo si así lo decidían. Este estudio se ajusta a la política de la Universidad Santo Tomás sobre el consentimiento ético y los estándares de cuidado de los animales.

\section{Resultados y Discusión}

Los valores de $\mathrm{Col}_{t}$ se distribuyeron entre 77 y $466 \mathrm{mg} / \mathrm{dl}$ y con una alta desviación estándar $( \pm 124.6 \mathrm{mg} / \mathrm{dl})$. Si se considera que los perros tenían una condición corporal $3 / 5$, que recibían alimento comercial balanceado y que eran adultos, la dispersión podría atribuirse a la inclusión de perros de ambos sexos (29 machos y 21 hembras), así como de varias razas y cruces. El mestizaje conlleva una alta variabilidad genética que puede influir en el perfil lipídico (Brenten et al., 2016). Cardoso et al. (2012) y Chang et al. (2016) señalan diferencias por grupo racial para diversas variables hematológicas y bioquímicas. Asimismo, Lawler et al. (2007) hacen referencias a las diferencias en el Labrador Retriever, raza incluida en el estudio, mientras que Brenten et al. (2016) encontraron un incremento del Col en hembras mayores de un año.

El promedio de $\mathrm{Col}_{\mathrm{t}}$ fue $291.8 \pm 62.0$ $\mathrm{mg} / \mathrm{dl}$ (promedio $\pm \mathrm{IC}_{95 \%}$ ) en los 18 perros del estudio (Cuadro 1), valor promedio que
Cuadro 1. Concentraciones de dopamina sérica (DA) ${ }^{1}$ en tres grupos de perros de la Región de Valparaíso, Chile, según su valor de colesterol total $\left(\mathrm{Col}_{t}\right)$

\begin{tabular}{|c|c|c|}
\hline & $\begin{array}{c}\text { Colt }_{\mathrm{t}} \\
(\mathrm{mg} / \mathrm{dl})\end{array}$ & $\begin{array}{c}\text { DA } \\
(\mu \mathrm{g} / \mathrm{ml})\end{array}$ \\
\hline $\begin{array}{l}\text { Valores de } \\
\text { referencia }\end{array}$ & $135-345$ & $30 \pm 1$ \\
\hline Grupo 1 & $152.5 \pm 46.1$ & $69.0 \pm 37.8$ \\
\hline Grupo 2 & $281.0 \pm 9.7$ & $68.1 \pm 20.5$ \\
\hline Grupo 3 & $441.8 \pm 19.3$ & $69.1 \pm 37.0$ \\
\hline Promedio & $291.8 \pm 62.0$ & $68.8 \pm 17.8$ \\
\hline
\end{tabular}

Cuadro 2. Pendientes del ajuste lineal realizada a la data correspondiente a cada grupo de perros según su contenido de colesterol sérico

\begin{tabular}{cc}
\hline Grupo & Pendiente del ajuste lineal \\
\hline I & 1.05 \\
II & 1.68 \\
III & 1.37 \\
\hline
\end{tabular}

I: 77-200; II: 250-300; III: 400-470 mg/dl

se encuentra dentro de lo aceptado para la especie (Wittwer, 2012), pero con varias muestras con resultados fuera de los rangos de referencia. Los valores de DA fueron de $68.8 \pm 17.8 \mu \mathrm{g} / \mathrm{ml}$ (promedio $\pm \mathrm{IC}_{95 \%}$ ), promedio superior al considerado como normal para la especie y cercano a lo esperable para una población con problemas de ansiedad (Riva et al., 2008) (Cuadro 1). Se realizó un análisis para descartar valores atípicos (outliers), dada la alta variabilidad natural de la molécula de DA, determinándose que no era necesario descartar algún resultado. 

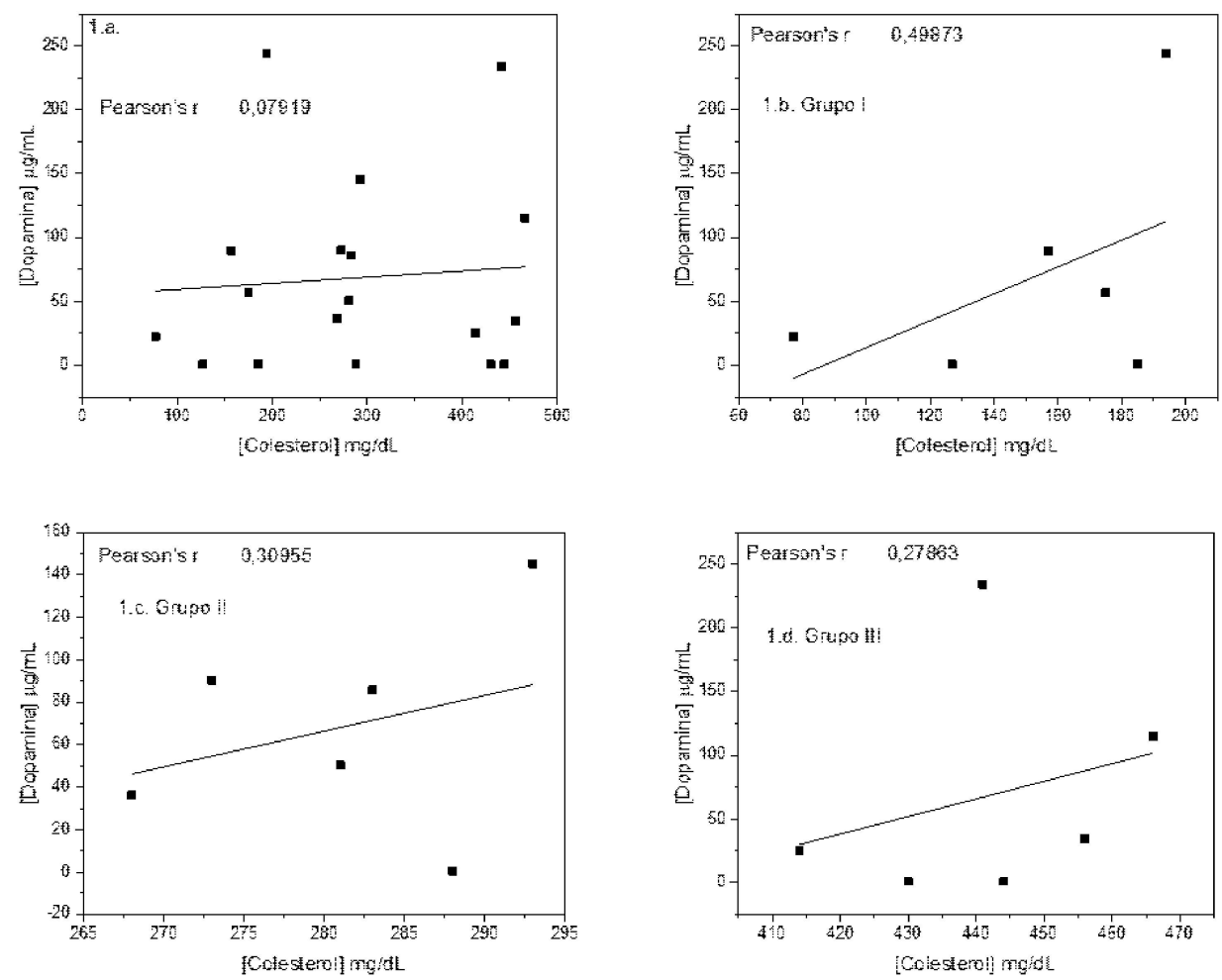

Figura 1. 1a. Asociación entre colesterol total $\left(\mathrm{Col}_{\mathrm{t}}\right)$ y dopamina sérica (DA) en los grupos en estudio $(\mathrm{n}=18) .1 \mathrm{~b}, 1 \mathrm{c}, 1 \mathrm{~d}$.: Correlación entre $\mathrm{Col}_{\mathrm{t}}$ y DA para los grupos I, II y II $(\mathrm{n}=6$ por grupo). I: 77-200; II: 250-300; III: 400-470 mg/dl de colesterol total

En la correlación entre el contenido de colesterol total $\left(\mathrm{Col}_{\mathrm{t}}\right)$ y DA sérico se observó una baja dependencia entre ambas variables, con un $\mathrm{r}=0.079$. Sin embargo, al analizar esta correlación para cada grupo se pudo observar una mayor dependencia en el grupo I; es decir, el grupo de perros con la menor concentración de colesterol $(\mathrm{r}=0.5)$.

Aun cuando no existen referencias entre el contenido de colesterol sérico y la concentración de DA en el perro, Hong y Amara (2010) propusieron una asociación entre el Col de membrana y la DA, donde a menor concentración de Col se tiene menor concentración de DA, ya que la actividad de transporte de sustrato de estos transportadores se inhibe, en general, por el agotamiento del Col
(Adkins et al., 2007; Foster et al., 2008; Hong y Amara, 2010). Si bien no hubo un control absoluto de la dieta recibida por los pacientes, los resultados que indican una menor correlación entre una alta concentración de Col plasmático con DA permiten suponer que el control de la dieta no jugaría un rol importante en este proceso.

Las asociaciones que fueron descritas entre estas variables por varios autores (Adkins et al., 2007; Foster et al., 2008; Hong y Amara, 2010) para humanos y ratas fueron relacionadas con la presencia del Transportador de Dopamina Plasmática (DAt), que corresponde a una proteína integral de membrana presináptica del sistema nervioso central (SNC). Dichos estudios demostraron que 
el Col puede modular la conformación del DAt y alterar la unión a DA en las especies estudiadas, teoría que no ha sido posible demostrar para el caso de los caninos.

En el análisis de las pendientes de las rectas de cada grupo de estudio se puede observar una mayor pendiente en el grupo II; es decir, aun cuando este grupo tiene un menor nivel de asociación entre las variables, pequeñas variaciones en el contenido de colesterol sérico aumentan el contenido de DA, o al menos, en mayor medida, al compararse con los grupos I y III.

A pesar de que las dos principales fuentes de colesterol celular se derivan de la síntesis de novo y de la captación de lipoproteínas plasmáticas, de que la mayoría del Col celular libre está presente en la membrana plasmática (Olsen et al., 2013) y de que para el aporte de colesterol corporal se estima en una proporción de $~ 70: 30$ entre la contribución de la síntesis de colesterol de novo y la ingesta dietética (Ikonen, 2008) y aunque el Col es el lípido con mayor importancia en la relación con la DA (Hong y Amara, 2010), esto probablemente varía considerablemente entre individuos. Esto depende de la constitución genética (eficacia en la producción de colesterol en comparación con la absorción) y el suministro de colesterol en la dieta (Ikonen, 2008).

Es posible que, debido a los factores mencionados, en el presente estudio no se haya logrado comprobar dicha asociación. Es probable que la medición de lípidos en sangre no haya dado cabida a otros metabolitos de los lípidos, de las cuales, tal vez, se podría haber obtenido más información; por ejemplo, en la medición de lipoproteínas que son responsables del transporte endógeno de $\mathrm{Col}$ (LDL-HDL) (Chung et al., 2004). Diversos estudios epidemiológicos y retrospectivos han mostrado una relación directa entre el colesterol total y el colesterol unido a estas lipoproteínas (C-LDL) con la morbilidad y mortalidad debida a causas cardiovasculares (Ascaso, 2010). El efecto concomitante en la inhibición de la enzima 3-hidroxi-3metilglutaril-coenzima A reductasa (HMGCoAR) y de la absorción de colesterol a nivel intestinal, mediante estatinas y ezetimiba, respectivamente, maximizan el descenso del C-LDL, relacionado con una reducción de las complicaciones cardiovasculares como la hipertensión, la ateroesclerosis y muerte por complicaciones coronarias (Ballantyne, 2002).

En investigaciones futuras se recomienda realizar un perfil lipídico completo, además de trabajar con tamaños poblacionales mayores, grupos más estandarizados, y tener en cuenta el factor edad, sexo y raza; esto último considerando que se han descrito diferentes fenotipos bioquímicos entre razas de perros (Cardoso et al., 2012; Brenten et al., 2016; Chang et al., 2016).

Chávez et al. (2013) encontraron un promedio de $62 \mu \mathrm{g} / \mathrm{mL}$ de DA plasmática en perros clasificados con algún grado de ansiedad, valor similar a los observados en el presente estudio. Coincidentemente, los niveles más altos de DA se observaban en pacientes con estados más complejos de ansiedad y, a su vez, con alteraciones de la conducta de tipo agresivo (principalmente por conflicto jerárquico e intraespecífico). Esto concuerda con lo planteado por Reisner (2002), quien dice que la ansiedad desempeña un rol importante en la génesis de la agresividad. En este estudio, los pacientes presentaron niveles de DA más cercanos a los de un perro ansioso que a los de un estándar para la especie (Riva et al., 2008). Frente a estos resultados, surge la duda si los perros de la zona de Valparaíso tienen niveles de DA más altos o si son animales más ansiosos debido a la forma de manejo (Chávez et al., 2012).

\section{Conclusiones}

- Existe una amplia distribución de valores en colesterol total $\left(\mathrm{COL}_{\mathrm{t}}\right.$ ) y dopamina (DA) sérica en perros, con una baja correlación entre estas variables. 
- Se encontró una mayor correlación entre $\mathrm{Col}_{\mathrm{t}}$ y DA para bajas concentraciones de $\mathrm{Col}_{\mathrm{t}}(\mathrm{r}=0.50)$.

\section{Literatura Citada}

1. Adkins EM, Samuvel DJ, Fog JU, Eriksen J, Jayanthi L, Vaegter C, et al. 2007. Membrane mobility and microdomain association of the dopamine transporter studied with fluorescence correlation spectroscopy and fluorescence recovery after photoblea-ching. Biochemistry 46: 10484-10497. doi: 10.1021/bi700429z

2. Allen J, Halverson-Tamboli RA, Rasenick M. 2007. Lipid raft microdomains and neurotransmitter signalling. Nat Rev Neurosci 8: 128-140. doi: 10.1038/nrn2059

3. Ascaso JF. 2010. Avances en el tratamiento de la hipercolesterolemia. Endocrinol Nutr 57: 210-219. doi: 10.1016/j.endonu.2010.03.008

4. Ballantyne C. 2002. Ezetimibe: efficacy and safety in clinical trials. Eur Heart $\mathrm{J}$ 4: J5-18. doi: 10.1016/S1520-765X(02)90077-5

5. Brenten T, Morris PJ, Salt C, Raila J, Kohn B, Schweigert FJ, Zentek J. 2016. Age-associated and breedassociated variations in haematological and biochemical variables in young Labrador Retriever and Miniature Schnauzer. Vet Rec Open 3(1): e000166. doi: 10.1136/vetreco-2015-000166

6. Brown D, London E. 1998. Functions of lipid rafts in biological membranes. Annu Rev Cell Dev Biol 14: 111-136. doi: 10.1146/annurev.cellbio.14.1.111

7. Butchbach M, Tian G, Guo H, Lin C. 2004. Association of excitatory amino acid transporters, especially EAAT2, with cholesterol-rich lipid raft microdomains: importance for excitatory amino acid transporter localization and function. $\mathrm{J}$ Biol Chem 279: 34388-34396. doi: 10.1074/jbc.M403938200
8. Cardoso JM, Fagnani R, Zaghi Cavalcante C, de Souza M, Júnior AZ, Fertonani L, Calesso JR, et al. 2012. Blood pressure, serum glucose, cholesterol, and triglycerides in dogs with different body scores. Vet Med Int. 2016: ID 8675283. doi: $10.1155 / 2016 / 8675283$; 2016:8675283

9. Chang Y, Hadox E, Szladovits B, Garden $O A$. 2016. Serum biochemical phenotypes in the domestic dog. PLoS One 11(2): e0149650. doi: 10.1371/ journal.pone. 0149650

10. Chávez G, Bustos C, Ubilla MJ. 2012. Frecuencia de trastornos conductuales en perros. Hospitales Vet 4: 119-124.

11. Chávez G, Dagnino P, Cuevas F, Opazo A, Marín MP. 2013. Correlación de los niveles de dopamina plasmática entre perros con distintos tipos de agresividad. Rev Med Vet 26: 9199. doi: 10.19052/mv.2635

12. Chung BH, Liang P, Doran S, Cho S, Franklin F. 2004. Postprandial chylomicrons: potent vehicles for transporting cholesterol from endogenous LDL+HDL and cell membranes to the liver via LCAT and CETP. J Lipid Res 45: 1242-1255. doi: 10.1194/ jlr.M300350-JLR200

13. Dodman N, Shuster L. 1998. Pshychopharmacology of animal behavior disorders. EEUU: Blackwell Science. 338 p.

14. Foster J, Adkins S, Lever J, Vaughan R. 2008. Phorbol ester induced trafficking-independent regulation and enhanced phosphorylation of the dopamine transporter associated with membrane rafts and cholesterol. J Neurochem 105: 1683-1699. doi: 10.1111/ j.1471-4159.2008.05262.x

15. Goodman A, Goodman L, Gilman A. 2007. Las bases farmacológicas de la terapéutica. $12^{\circ}$ ed. Colombia: McGrawHill. $2017 \mathrm{p}$.

16. Hong C, Amara G 2010. Membrane cholesterol modulates the outward facing conformation of the dopamine transport- 
er and alters cocaine binding. J Biol Chem 285: 32616-32626. doi: 10.1074/ jbc.M110.150565

17. Ikonen E. 2008. Cellular cholesterol trafficking and compartmentalization. Nat Rev Mol Cell Biol 9: 125-138. doi: $10.1038 / \mathrm{nrm} 2336$

18. Jackson D, Westlind-Danielsson A. 1994. Dopamine receptors: molecular biology, biochemistry and behavioral aspects. Pharmacol Ther 64: 291-370. doi: 10.1016/0163-7258(94)90041-8

19. Jayanthi L, Samuvel D, Ramamoorthy S. 2004. Regulated internalization and phosphorylation of the native norepinephrine transporter in response to phorbol esters. Evidence for localization in lipid rafts and lipid raft-mediated internalization. J Biol Chem 279: 1931519326. doi: 10.1074/jbc.M311172200

20. Kirk R, Bonagura J. 2001. Terapéutica veterinaria de pequeños animales. $13^{\circ}$ ed. Madrid, España: McGraw-Hill. 756 p.

21. Lawler DF, Ballam JM, Meadows R, Larson BT, Li Q, Stowe HD, Kealy RD. 2007. Influence of lifetime food restriction on physiological variables in Labrador Retriever dogs. Exp Gerontol 42: 204-214. doi: 10.1016/j.exger.2006.09.010

22. Lindsay S. 2000. Handbook of applied dog behavior and training: adaptation and learning. Vol 1. Iowa, USA: Blackwell Publishing. $386 \mathrm{p}$.

23. Magnani F, Tate C, Wynne S, Williams C, Haase J. 2004. Partitioning of the serotonin transporter into lipid microdomains modulates transport of serotonin. J Biol Chem 279: 3877038778. doi: 10.1074/jbc.M400831200

24. Maldonado O, Ramírez I, García JR, Ceballos G, Méndez E. 2012. Colesterol: función biológica e implica-ciones médicas. Rev Mex Cienc Farm 43: 7-22.
25. Miczek K, Fish E, de Bold J, De Almeida R. 2002. Social and neural determinants of aggressive behavior: pharmacotherapeutic targets at serotonin, dopamine and $\gamma$-aminobutyric acid systems. Psychopharmacology 163: 434458. doi: 10.1007/s00213-002-1139-6

26. Murray R, Granner D, Mayes $P$, Rodwell V. 2003. Harper's illustrated biochemistry. $26^{\text {th }}$ ed. New York, USA: McGraw-Hill. 702 p.

27. Núñez E, Alonso-Torres P, Fornés A, Aragón C, López-Corcuera B. 2008. The neuronal glycine transporter GLYT2 associates with membrane rafts: functional modulation by lipid environment. J Neurochem 105: 20802090. doi: 10.1111/j.1471-4159.2008.05292.x

28. Olsen BN, Bielska AA, Lee T, Daily $M D$, Covey DF, Schlesinger PH, Baker NA, Ory DS. 2013. The structural basis of cholesterol accessibility in membranes. Biophys $\mathrm{J}$ 105: 1838-1847. doi: 10.1016/ j.bpj.2013.08.042

29. Pereira H. 2017. The importance of cholesterol in psychopathology: a review of recent contributions. Indian J Psychol Med 39: 109-113. doi: 10.4103/02537176.203117

30. Reisner IR. 2002. An overview of aggression. En: BSAVA Manual of canine and feline behavioural medicine. $2^{\text {nd }}$ ed. Gloucester, UK: British Small Animal Veterinary Association. p 181194.

31. Riva J, Bondiolotti G, Michelazzi M, Verga M, Carenzi C. 2008. Anxiety related behavioural disorders and neurotransmitters in dogs. Appl Anim Behav Sci 114: 168-181. doi: 10.1016/ j.applanim.2008.01.020 
32. Pentürk S, Yalçin E. 2003. Hypocholesterolaemia in dogs with dominance aggression. J Vet Med A Physiol Pathol Clin Med 50: 339-342. doi: 10.1046/ j.1439-0442.2003.00556.x

33. Siever L. 2008. Neurobiología de la agresividad y la violencia. Anuario Psicol Jurídica 26: 60-69. doi: 10.1016/ j.apj.2016.03.001
34. Simons K, Ikonen E. 1997. Functional rafts in cell membranes. Nature 387: 569572. doi: $10.1038 / 42408$

35. Vega-Badillo J. 2017. Alteraciones en la homeostasis del colesterol hepático y sus implicaciones en la esteatohepatitis no alcohólica. TIP 20: 50-65. doi: 10.1016/j.recqb.2016.11.005

36. Wittwer F. 2012. Manual de patología clínica veterinaria. $2^{\circ}$ ed. Valdivia, Chile: Imprenta América. 176 p. 